\title{
Korean nova records in A.D. 1073 and A.D. 1074: R Aquarii
}

\author{
Hong-Jin Yang ${ }^{1}$, Myeong-Gu Park ${ }^{2}$, Se-Hyung Cho ${ }^{1}$, and Changbom Park ${ }^{3}$ \\ 1 Korea Astronomy and Space Science Institute, 61-1 Hwaam, Yuseong, Daejeon 305-348, Korea \\ e-mail: [hjyang;cho]@kasi.re.kr \\ ${ }^{2}$ Department of Astronomy and Atmospheric Sciences, Kyungpook National University, Daegu 702-701, Korea \\ e-mail: mgp@knu.ac.kr \\ 3 School of Physics, Korea Institute for Advanced Study, Seoul 130-722, Korea \\ e-mail: cbp@kias.re.kr
}

Received 30 November 2004 / Accepted 31 December 2004

\begin{abstract}
R Aqr is known to be a symbiotic binary system with an associated extended emission nebula, possibly produced by a historic outburst. To find the associated historic records, we searched for and compiled all Guest Star and Peculiar Star records in three Korean official history books that cover almost two thousand years, Samguksagi, Goryeosa, Joseonwangjosillok. In addition to the record of A.D. 1073, previously noted by Li (1985, Chin. Astron. Astrophys., 9, 322), we have found in Goryeosa another candidate record of A.D. 1074, which has the same positional description as that of A.D. 1073 with an additional brightness description. We examined various aspects of the two records and conclude that they both are likely to be the records of outburst of R Aqr. This means that there were two successive outbursts in A.D. 1073 and in A.D. 1074, separated by approximately one year. Based on these records, we estimate the distance to R Aqr to be $273 \mathrm{pc}$ if the expansion of the nebula has been at a constant rate. The brightness record of A.D. 1074 corresponds to the absolute magnitude at outburst of $M_{\text {outburst }}=-6.2^{\mathrm{m}} \sim-5.2^{\mathrm{m}}$ at this distance. The two Korean records associated with R Aqr may provide astronomically meaningful constraints to the outburst model of R Aqr and the formative process of its nebulosity.
\end{abstract}

Key words. stars: binaries: symbiotic - ISM: planetary nebulae: general - history and philosophy of astronomy

\section{Introduction}

$\mathrm{R}$ Aquarii is one of the more enigmatic astronomical objects that has attracted investigators for many decades. R Aqr, located at RA $23^{\mathrm{h}} 43.8^{\mathrm{m}}$, Dec $-15^{\circ} 17.0^{\prime}(\mathrm{J} 2000.0)$, is the nearest known symbiotic binary system at about 250 pc distance (Whitelock 1987) and also has an astrophysical jet (Paresce et al. 1991). The primary is a Mira variable of spectral type M7e with a pulsational period of 386.83 days whose variable nature was first noted by Harding (1816). The secondary is generally believed to be a white dwarf (Henny \& Dyson 1992). R Aqr is known to be associated with an extended emission nebula (Herbig 1965). The complex nebula which surrounds the central star in a remarkable point-symmetric arrangement consists of two main components, the inner and outer nebulosities, both discovered by Lampland (1923a,b). The outer nebulosity is an oval-shaped formation of about $90^{\prime \prime}$ extent composed of two intersecting, well-defined arcs and shape of a double convex lens oriented nearly east and west (Solf \& Ulrich 1985). Outward motions in the outer nebulosity were suspected by Hubble (1940, 1943). Baade (1944) detected outward motions of the nebula from comparison of plates taken 16 years apart and concluded that, assuming a constant rate of expansion, the nebula was $600 \mathrm{yr}$ old. The inner nebulosity shows a wedgeshaped structure, that reaches about $30^{\prime \prime}$ extent and appears to extend north and south, perpendicular to the orientation of the outer nebulosity (Solf \& Ulrich 1985). Two nebulosities have dense equatorial ring structure. Equatorial velocities of 55 and $32 \mathrm{~km} \mathrm{~s}^{-1}$ are derived from the outer and inner shell-like nebulosities, respectively, and the expansion rates combined with tangential motions from the literature yield a distance of about 180 pc for both shells (Solf \& Ulrich 1985). Solf \& Ulrich (1985) argued that the uniformity observed in the kinematics of each shell indicates that the shells can be attributed to two major ejection events, 650 and 190 years ago, and that further outbursts within the last centuries are unlikely.

A new feature within the inner nebulosity appeared between 1970 and 1977. A spike or jet has appeared as a protrusion from the central star toward the northeast (Hollis et al. 1989). The jet has been observed in the optical (Wallerstein \& Greenstein 1980; Herbig 1980), in the radio at 2 and $6 \mathrm{~cm}$ with the VLA (Sopka et al. 1982; Kafatos \& Michalitsianos 1983), and in the far UV with the International Ultraviolet Explorer (IUE). The jet may be powering the R Aqr nebula that radiates more than $5 \times 10^{44} \mathrm{erg} \mathrm{yr}^{-1}$ in the Balmer and Lyman continua and emission lines (Kafatos \& Michalitsianos 1982).

Kafatos \& Michalitsianos (1982) and Li (1985) separately suggested historical records related to the outburst of R Aqr. Kafatos \& Michalitsianos (1982) proposed that a 
historical Japanese astronomical record of a nova outburst in A.D. 930 might be associated with R Aqr, which formed the outer extended nebulosity. However, Li (1985) pointed out the erroneous identification of the Japanese record of A.D. 930 for R Aqr. The Japanese record was based on records in Meigetuki and Ichidaiyouki, reproduced later in Japanese Ancient Astronomical Records (Kanda 1935). The record in Ichidaiyouki reads: a Guest Star enters Urim (one of the oriental constellations), begins to move westwards in Urim. $\mathrm{Li}$ (1985) concluded that in view of the words enter and move westwards, the event of A.D. 930 should be considered as that of a comet rather than a nova. We confirm this because the record of 930 is actually classified as a comet in Japanese Ancient Astronomical Records (Kanda 1935). Li also suggested a Korean astronomical record of A.D. 1073 as a description of nova outburst of R Aqr. The Korean record of 1073 reads simply: a Guest Star is seen south of DongByeok (another oriental constellation, located in the north of Urim).

Historical nova observations have been recorded in many countries such as Korea, China and Japan as well as various European and Arabian countries. Lundmark (1921) collected many observations of historical novae and compiled a cata$\log$ that includes 60 suspected nova records before or during 19th century. Most of Lundmark's (1921) catalog is based on Chinese records translated by Biot (1846) and Williams (1871). Hsi (1958) revised the previous list of historical Chinese New Star catalog of 90 suspected novae. While Korea has abundant suspected historical nova records, they have not been compiled systematically from the original sources in the previous literatures. Hence, we searched for and compiled all Guest Star and Peculiar Star records in the original official Korean chronicles. We have found two records that could be associated with the outburst of R Aqr: records of A.D. 1073 and 1074 (Cho et al. 1999). The record of A.D. 1074 that even has brightness information has never been mentioned as a candidate record of outburst of R Aqr.

\section{Korean records of Guest Star and Peculiar Star}

Korea has abundant and homogeneous historical astronomical records in the three major history books: Samguksagi ${ }^{1}$ (The History of the Three Kingdoms), Goryeosa (The History of the Goryeo Dynasty), and Joseonwangjosillok (The Annals of the Joseon Dynasty). They are deemed to be official history books because the subsequent dynasties or the reigning monarchy itself oversaw the writing based on previous history books. Samguksagi covers the period from 57 B.C. to A.D. 935: the period of Three Kingdoms, namely Silla, Goguryeo, and Baekje. It contains total of 236 records of solar eclipses, comets, meteors, planet motions and so forth. Goryeosa covers the Goryeo dynasty from A.D. 918 to A.D. 1392. It is a well-arranged history book divided into several chapters by contents, and records are listed in chronological order. Joseonwangjosillok (Sillok, hereafter), the most recent and the

\footnotetext{
1 All Korean historical names in this paper follow the notation used by the Korean government. Corresponding names in Chinese characters are given in the Appendix A.
}

most extensive official chronicles, covers Joseon dynasty from A.D. 1392 to A.D. 1910. In addition to these official history books, Jeungbomunheonbigo, a collection of the rearranged records compiled from the official Korean chronicles, also has abundant astronomical records divided into several chapters by contents.

Korean historical astronomical records are in general described as one of several classes of astronomical phenomena such as solar and lunar eclipses, comets, meteors, planet motions and so forth. Among these records, some of Guest Star and Peculiar Star records are possibly related with outburst of stars. Literally, Guest Star means a new star. The first Korean Guest Star record appears in A.D. 85 and the second in A.D. 154, both in Samguksagi. More Guest Star records appear continuously up to A.D. 1770 . We have compiled all Guest Star records in Table 1 from the Samguksagi, Goryeosa, Sillok, and Jeungbomunheonbigo, covering almost two thousand years. We found 39 of them. However, two records of A.D. 1600 seem to be duplicate of the same record in 1604 . The two records in 1600 appear only in Jeungbomunheonbigo which is a encyclopedia, and sometimes contains inaccurate records, particularly in date. Furthermore, the descriptions of two records in 1600 are the same as that in 1604.

A Guest Star in Korean history books mainly represents a nova or a comet. However, when the movements of a Guest Star are subsequently observed, the Guest Star is recorded as a comet afterwards. For instance, one record in Goryeosa on 4 April 1066 reads: a star, as big as the Moon, appears to the northwest, and suddenly becomes a comet.

Sometimes Guest Star records have descriptions of position and size. Since the position of Guest Star is described based on the oriental constellation or relative to planets or moon, we can convert the position into the corresponding modern constellation. Ahn et al. (1996) and Park (1998) have identified most of oriental constellations with associated modern constellations. We have found five Guest Star records during the Three Kingdoms period. Two of them, records in A.D. 85 and A.D. 867/868, have positional information but the locations do not coincide with those of R Aqr. Goryeosa has seven Guest Star records. One in 1363 is recorded as a Guest Star, but it seems to be related with meteor shower. The description reads: seven Guest stars appear simultaneously and three small stars fight against each other. All records have positional information except the record of 1065. Among them, two records in 1073 and 1074 seem to be related with R Aqr based on estimated location and actual visibility on the recorded night. The record of 1073 has been suggested to be associated with the outburst of R Aqr by Li (1985). In addition to the record of 1073 we have found another new candidate record for the outburst of R Aqr. The positional information of the record of 1074 is the same as that of 1073, but it has an additional description of brightness. The description of records of 1073 and 1074 are shown in Table 3.

Joseon dynasty has roughly 340 Guest Star records in total, describing about 25 independent Guest Star events. Most of them are the observations of the well-known Kepler's supernova of 1604. Twelve of them seem to be related with comets because the descriptions include the movement of the Guest 
Table 1. Historical Korean Guest Star records compiled from the three Korean history books (Samguksagi, Goryeosa, and Joseonwangjosillok) and an encyclopedia (Jeungbomunheonbigo).

\begin{tabular}{|c|c|c|c|c|c|}
\hline Date of observation $^{a b}$ & $\mathrm{JD}^{c}$ & $\begin{array}{l}\text { Associated }^{d} \\
\text { constellations }^{2}\end{array}$ & $\operatorname{Ref}^{e}$ & $\begin{array}{c}\text { Duration }^{f} \\
\text { (days) }\end{array}$ & Note $^{g}$ \\
\hline - May/June 85 & 1752255 & Dra/UMi/Cep & $\mathrm{S}, \mathrm{M}$ & & \\
\hline - Jan. 154 & 1777321 & - & $\mathrm{S}, \mathrm{M}$ & & \\
\hline - Oct./Nov. 299 & 1830571 & - & $\mathrm{S}, \mathrm{M}$ & & \\
\hline--662 & - & - & $\mathrm{M}$ & & \\
\hline - Dec./Jan. 867/868 & 2038094 & $\mathrm{Lib} / \mathrm{Sco} / \mathrm{Sgr}$ & $\mathrm{S}, \mathrm{M}$ & & \\
\hline 01 Aug. 1065 & 2110262 & - & $\mathrm{G}, \mathrm{M}$ & & \\
\hline 10 Sep. 1073 & 2113224 & Aqr/Psc/Cap & $\mathrm{G}, \mathrm{M}$ & & \\
\hline 19 Aug. 1074 & 2113567 & $\mathrm{Aqr} / \mathrm{Psc} / \mathrm{Cap}$ & $\mathrm{G}, \mathrm{M}$ & & \\
\hline 10 Aug. 1163 & 2146065 & Oph & $\mathrm{G}, \mathrm{M}$ & & \\
\hline 03 May 1356 & 2216460 & Tau & $\mathrm{G}, \mathrm{M}$ & & \\
\hline 09 June 1363 & 2219053 & - & $\mathrm{G}, \mathrm{M}$ & & Shower \\
\hline 22 May 1391 & 2229262 & Dra/UMi/Cep & $\mathrm{G}, \mathrm{M}$ & & \\
\hline 05 Jan. 1399 & 2232047 & Oph & $\mathrm{J}, \mathrm{M}$ & & \\
\hline 11 Mar. 1437 & 2245992 & Sco & $\mathrm{J}, \mathrm{M}$ & 14 & \\
\hline 24 Aug. 1499 & 2268803 & Dra/UMi & $\mathrm{J}, \mathrm{M}$ & 4 & Comet \\
\hline 06 Nov. 1572 & 2295541 & Cas & $\mathrm{J}, \mathrm{M}$ & & \\
\hline 23 Nov. 1592 & 2302853 & Cet & $\mathrm{J}, \mathrm{M}$ & 457 & \\
\hline 30 Nov. 1592 & 2302860 & Cas & $\mathrm{J}, \mathrm{M}$ & 118 & \\
\hline 04 Dec. 1592 & 2302863 & Cas & $\mathrm{J}, \mathrm{M}$ & 115 & \\
\hline 12 Dec. 1592 & 2302872 & And? & $\mathrm{J}$ & & \\
\hline 18 Jan. 1593 & 2302909 & And? & $\mathrm{J}$ & & $?$ \\
\hline - Nov./Dec. 1600 & 2305782 & Sco & M & & Typo $^{h}$ ? \\
\hline - Dec./Jan. 1600/1601 & 2305813 & Sco & $\mathrm{M}$ & & Typo? \\
\hline 13 Oct. 1604 & 2307195 & Sco & $\mathrm{J}, \mathrm{M}$ & 201 & \\
\hline 27 Oct. 1639 & 2319992 & Lep & $\mathrm{J}$ & 3 & Comet \\
\hline 04 Feb. 1661 & 2327763 & Del/Aql & $\mathrm{J}$ & 5 & Comet \\
\hline 13 Dec. 1661 & 2328075 & Aqr & $\mathrm{J}, \mathrm{M}$ & 20 & Comet \\
\hline 20 Nov. 1684 & 2336453 & Vir & M & & \\
\hline 22 Apr. 1702 & 2342814 & $\mathrm{Sco} / \mathrm{Sgr}$ & $\mathrm{J}, \mathrm{M}$ & 9 & Comet \\
\hline 13 Feb. 1737 & 2355530 & Peg/Aqr/Cep & $\mathrm{J}$ & & \\
\hline 06 Mar. 1742 & 2357377 & $\mathrm{Sgr} / \mathrm{Cap}$ & $\mathrm{J}$ & 2 & Comet \\
\hline 13 Feb. 1743 & 2357721 & Crv-UMa-Crt & $\mathrm{J}, \mathrm{M}$ & 9 & Comet \\
\hline 06 Jan. 1744 & 2358048 & Peg/And/Aqr & $\mathrm{J}$ & 2 & Comet? \\
\hline 17 Nov. 1744 & 2358364 & Vir & $\mathrm{J}$ & 2 & Comet \\
\hline 02 May. 1748 & 2359626 & Peg & $\mathrm{J}$ & & \\
\hline 19 Dec. 1759 & 2363874 & Cet & $\mathrm{J}$ & & \\
\hline 08 Jan. 1760 & 2363894 & Cma-Cet & $\mathrm{J}, \mathrm{M}$ & 14 & Comet \\
\hline 09 Feb. 1760 & 2363926 & Leo & $\mathrm{J}, \mathrm{M}$ & 11 & Comet \\
\hline 29 June 1770 & 2367719 & Oph-Cap-Sgr & $\mathrm{J}, \mathrm{M}$ & 4 & Comet \\
\hline
\end{tabular}

${ }^{a}$ Julian calendar for dates prior to 4 Oct. 1582, Gregorian calendar after 15 Oct. 1582.

${ }^{b}$ Records with no month or day information are noted by - .

${ }^{c}$ The record with no day information is set to be the fifteenth day of recorded lunar month.

${ }^{d}$ Estimated position over the modern constellations deduced from original description on position described as oriental constellations, a planet or the moon.

${ }^{e} \mathrm{~S}$ means Samguksagi, G Goryeosa, J Joseonwangjosillok, and M Jeungbomunheonbigo.

${ }^{f}$ Observational duration for the same guest star.

${ }^{g}$ Estimated astronomical phenomenon deduced from original description.

${ }^{h}$ Typographical error.

Stars. These are marked as a Comet in the seventh column in Table 1. However, we cannot be sure about the real nature of the object for every record. We also identified the location of all Guest Star events with associated constellations in Table 1. Among these records, only one record of January 1744 in Sillok has a possibility to be associated with R Aqr, it reads simply: a Guest Star appears in the region of DongByeok and its appearance resembles a comet. The record of January 1744 also appears in Chinese and Japanese chronicles, but it is described as a comet in the two chronicles. Another record of 1737 is also related with the constellation of Aquarius, but estimated location of the record is apart from that of R Aqr.

Supernova is also recorded as a Guest Star in the Korean history books. Since Koreans believed that a special 
Table 2. Historical Korean Peculiar Star records compiled from the three Korean representative history books (Samguksagi, Goryeosa, and Joseonwangjosillok) and Jeungbomunheonbigo. All notations in this table are the same as in Table 1.

\begin{tabular}{lclcc}
\hline \hline $\begin{array}{l}\text { Date of } \\
\text { observation }\end{array}$ & JD & $\begin{array}{l}\text { Associated } \\
\text { constellations }\end{array}$ & Ref. & Note \\
\hline--673 & - & - & S & \\
--744 & - & - & S, M & \\
03 Oct. 932 & 2061747 & - & G, M & \\
31 May 1021 & 2094129 & Com/Vir & G, M & \\
04 Oct. 1031 & 2097907 & Cnc & G, M & \\
25 Oct. 1063 & 2109616 & Oph & G, M & \\
14 Oct. 1072 & 2112893 & Peg & G, M & \\
04 Aug. 1082 & 2116474 & UMi & G, M & \\
11 Apr. 1176 & 2150693 & - & G, M & \\
05 Sep. 1382 & 2226081 & Leo & G, M & \\
10 Nov. 1577 & 2297371 & - & J & Comet \\
- 1625 & - & - & M & \\
- Aug./Sep. 1626 & 2315187 & - & M & \\
- Oct./Nov. 1627 & 2315612 & - & M & \\
- Feb./Mar. 1645 & 2321942 & Cnc & M & \\
04 Dec. 1707 & 2344866 & Del/Vul & J, M & \\
\hline & & & & \\
\hline
\end{tabular}

astronomical event hints the future, Guest Stars generally were recorded carefully. Although we do not find in Goryeosa the record of Crab supernova in 1054, while China and Japan have records of Crab supernova, it could have been caused by political disorder in the early Goryeo dynasty, because not a single astronomical record appears in Goryeosa for the whole year of 1054. Meanwhile, two supernova records of Tycho's and Kepler's are carefully recorded as Guest Stars in Sillok. Tycho's supernova was discovered by Koreans on 6 November 1572 and sighted two days later by Chinese. Kepler's supernova is also recorded from 13 October 1604 to 23 April 1605 in Sillok as a Guest Star with details such as size, angular distance from Polaris, color and so forth.

In addition to Guest Star records, another class of records that can be related with nova or cataclysmic phenomenon are Peculiar Star records. We also have compiled and listed all Peculiar Star records from Samguksagi, Goryeosa, Sillok, and Jeungbomunheonbigo. Some of them also have positional descriptions. We have listed the Peculiar Star records in Table 2. However, we have not found any adequate record to be related with R Aqr from the Peculiar Star records in Table 2.

\section{R Aqr and Guest Star of A.D. 1073 and 1074}

\subsection{Guest Star Records of A.D. 1073 and 1074}

Among all Korean historical Guest Star and Peculiar Star records listed in Tables 1 and 2, we have found two most relevant records in Goryeosa (also in Jeungbomunheonbigo), those of A.D. 1073 and 1074, that can be related with outburst of R Aqr. Figure 1 shows the original text of the two Guest Star records of A.D. 1073 and 1074 in Goryeosa. While the two records are separated by one year interval, the locations are described in the same way: a Guest Star is seen at the south of DongByeok. DongByeok is one of the 28 oriental
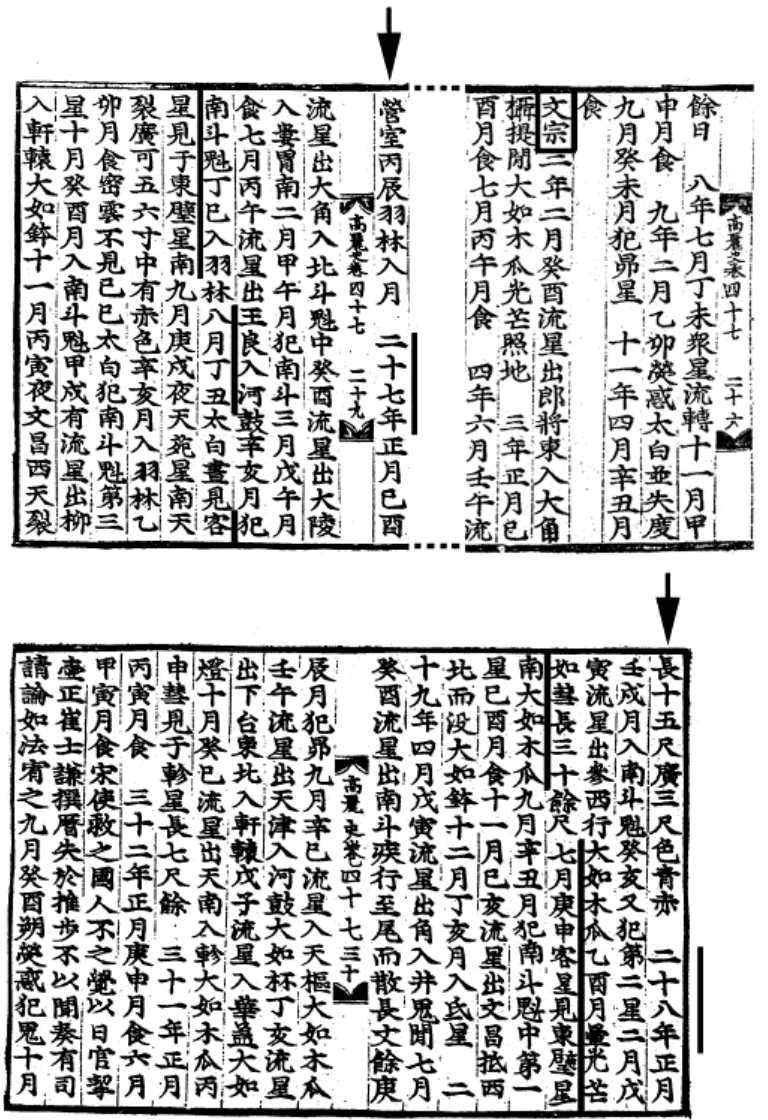

Fig. 1. The original two records of guest star on September 10, 1073 and August 19, 1074. The record of A.D. 1073 (in the upper box) reads as A Guest Star is seen south of DongByeok, and the record of A.D. 1074 (in the lower box) reads as A Guest Star is seen south of DongByeok and its size is similar to that of Mokgwa. DongByeok is one of the ancient oriental constellations and corresponds to $\alpha$ And and $\gamma$ Peg. Mokgwa is a quince and its size is similar to that of orange.

constellations (also known as 28 lunar lodges), which consists of $\alpha$ And and $\gamma$ Peg (Ahn et al. 1996; Park 1998). Although the two records of 1073 and 1074 are described in the same way, the dates of two records, recorded in lunar calendar with 60 cyclical days, are different. Moreover, a sighting of Venus at daylight was also recorded along with the Guest Star record of 1073 for the same day. The apparent magnitude of Venus at 10 September 1073 was $-4.19^{\mathrm{m}}$ whereas the mean apparent magnitude of Venus is $-3.19^{\mathrm{m}}$. These strongly support that the records of 1073 and 1074 are real and independent.

Also, notable is that the record of 1074 includes the size information of the Guest Star. The size of the Guest Star is recorded as a Mokgwa. Mokgwa is a Korean quince, the size of which is similar to that of the orange. Mokgwa (hereafter, quince) is often used to represent the size, i.e. brightness, of astronomical objects or phenomena in Korean history books. For instance, 91 meteor and 2 comet records in Goryeosa are described having the size of quince.

The two records of Guest Star in 1073 and 1074 appear only in Korean history books. We cannot find similar records for the same period either in General Compilation of Chinese 


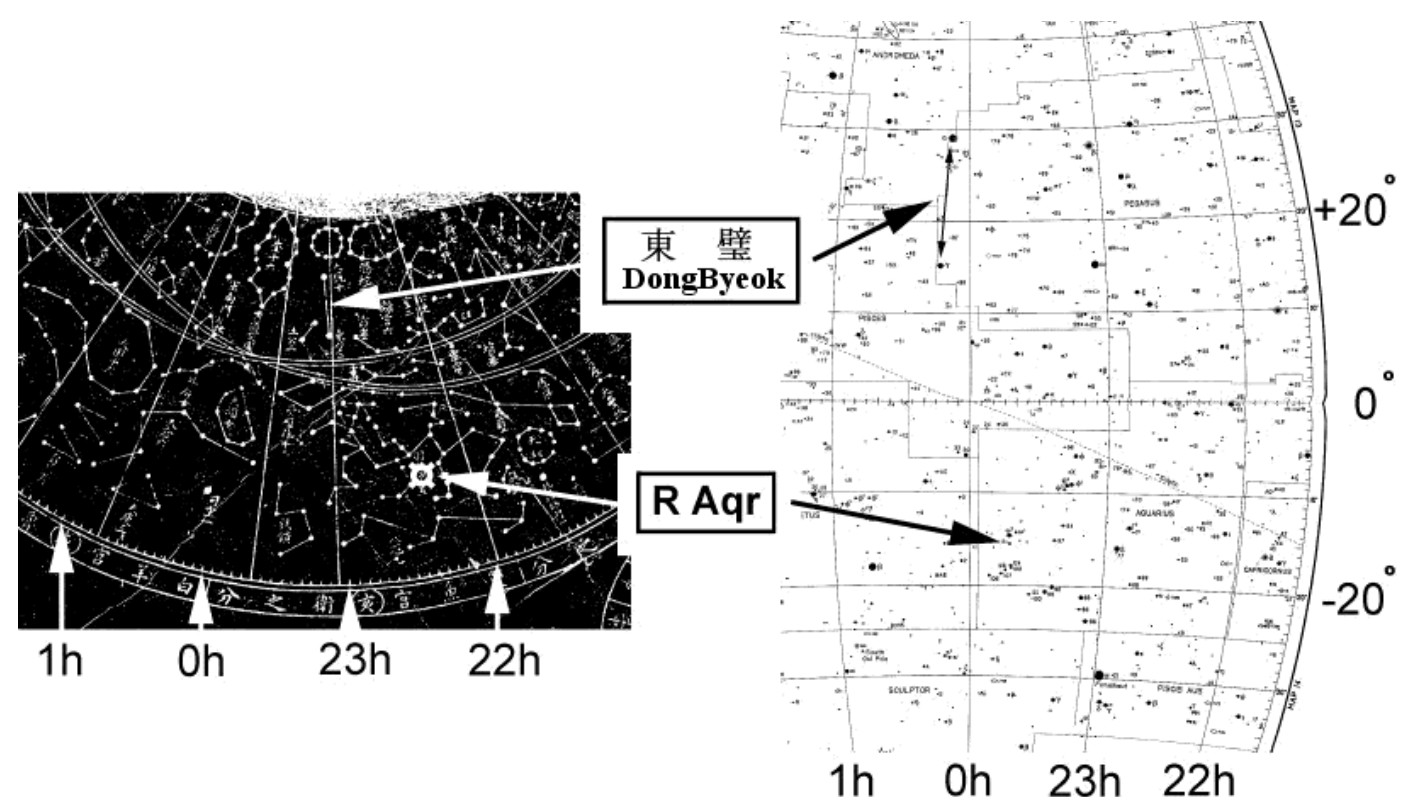

Fig. 2. The position of R Aquarii and DongByeok. The sky map on the left, which is a part of Cheonsangyeolchabunyajido, a Korean sky map carved in stone in A.D. 1395, shows the position of DongByeok and a corresponding position of R Aqr. The sky map on the right shows a position of R Aqr and the position of corresponding DongByeok, $\alpha$ And and $\gamma$ Peg, in modern celestial coordinates.

Table 3. Guest star records of A.D. 1073 and 1074.

\begin{tabular}{cll}
\hline \hline $\begin{array}{c}\text { Date of observation } \\
\text { (JD) }\end{array}$ & Original Text & Text description \\
\hline $\begin{array}{c}\text { 10 Sep. 1073 } \\
(2113224)\end{array}$ & $\begin{array}{l}\text { 文宗二十七年八月丁丑 } \\
\text { 客星見于東璧星南 }\end{array}$ & $\begin{array}{l}\text { A guest star appeared at the } \\
\text { south of DongByeok (東璧) }\end{array}$ \\
$\begin{array}{cll}\text { 19 Aug. 1074 } \\
(2113567)\end{array}$ & $\begin{array}{l}\text { 文宗二十八年七月庚辛 } \\
\text { 客星見東璧星南大如木瓜 }\end{array}$ & $\begin{array}{l}\text { A guest star, as big as a Mokgwa (木瓜 })^{c} \\
\text { appeared at the south of DongByeok }\end{array}$ \\
\hline
\end{tabular}

a Julian calendar for date

${ }^{b}$ One of the 28 oriental constellations, which consists of $\alpha$ And and $\gamma$ Peg

c A Korean quince with size similar to that of orange

Ancient Astronomical Records (Beijing Observatory 1988) or in Japanese Ancient Astronomical records (Kanda 1935).

\subsection{Locations and brightness}

Li (1985) suggested the historical Korean record of A.D. 1073 as a record of nova outburst of $\mathrm{R} \mathrm{Aqr.} \mathrm{Li,} \mathrm{however,} \mathrm{did} \mathrm{not}$ discuss the location of the Guest Star in 1073 relative to that of R Aqr.

Oriental astronomers divided the visible part of the heavens into 31 sections, 28 of which have been termed stellar divisions, and generally denoted by the asterism, forming the central or principal part of the division. Twenty-eight of them, each of which generally consists of several constellations, are located along the zodiac, whereas three of them, the Three Domains, occupy the central parts of celestial northern pole. The name of each division is also used for the representative constellation within it. We simply call these 28 divisions as 28 oriental constellations, hereafter. The 28 oriental constellations are irregular in their extent, both from north to south and from east to west. Figure 2 shows the location of R Aqr and constellation of DongByeok as appeared in Cheonsangyeolchabunyajido, the Korean sky map carved in stone in A.D. 1395, and in modern sky map. In terms of the oriental sky map, R Aqr is located in Urim, a constellation located in the south of DongByeok that is one of the 28 oriental constellations. Urim consists of 45 stars and occupies the region of RA $20^{\mathrm{h}} 30^{\mathrm{m}} \sim 23^{h} 20^{m}$, Dec $-20^{\circ} \sim 0^{\circ}$. As already mentioned, the two Guest Stars are recorded as seen in the south of DongByeok. Since it is essential to compare the locations in two records with that of $\mathrm{R} \mathrm{Aqr}$, we made some pertinent investigations.

First, we checked the recent novae, supernovae, and cataclysmic variable stars within $40^{\circ} \times 40^{\circ}$ in the south of DongByeok that would cover the region from $\gamma$ Peg to the horizon at the time of $\gamma$ Peg's transit. We have not found any candidate nova or supernova. The frequency of the nova outbursts brighter than 2nd mag. recorded during the past one hundred years is estimated to be about 9 novae per century in Our Galaxy (Payne-Gaposchkin 1964). The probability of finding a random nova within $40^{\circ} \times 40^{\circ}$ during the past one thousand years is only about 0.32 (Kafatos \& Michalitsianos 1982). Hence, the probability that the outburst of R Aqr is not 
recorded and the Guest Stars of 1073 and 1074 are the records of yet another nova or supernova is even smaller.

Second, we examined why the location of Guest Star is described as south of DongByeok instead of within Urim, the corresponding oriental constellation that encompasses R Aqr. We first searched recording formats of historical Guest Star records. Although we found no more Guest Star record with positional description in Goryeosa, we noted that the location of Guest Stars in Korean and Chinese chronicles are generally described with reference to the 28 oriental constellations such as DongByeok, which would have more significant astrological meanings than minor constellations such as Urim. For example, Guest Star records of A.D. 1604 A.D. 1605, Kepler's supernova, describe the location by the angular distance from the 28 oriental constellations and Polaris instead of the nearby minor constellations. And most Guest Star records in other official Korean history books have positional information based on the 28 oriental constellations. We also investigated the recording formats of the twelve Chinese Guest Star records from A.D. 1000 to A.D. 1300. Ten of them describe the location based on the 28 oriental constellations. In particular, the records of A.D. 1054, Crab supernova, appear independently in the four Chinese history books. Only one history book describes the location based on an ordinary star, whereas the positions in two history books are based on one of the 28 oriental constellations, which is 30 degrees away from the Crab supernova. So we believe that the Guest Star records of 1073 and 1074 followed the astronomical tradition in Korean and Chinese chronicles that described their celestial positions based on the 28 oriental constellations where the event instead of nearby minor constellations.

Moreover, it is quite likely that a brighter star was used as a reference when the location of a new or peculiar object was recorded. Magnitudes of stars in DongByeok ( $\alpha$ And: $2.06^{\mathrm{m}}$, $\gamma$ Peg: $\left.2.83^{\mathrm{m}}\right)$ are brighter than those in Urim $\left(3.27^{\mathrm{m}} \sim 5.08^{\mathrm{m}}\right)$. Furthermore, when R Aqr passes the transit, Urim would be located at about +27 degrees above the horizon while DongByeok is located at about +57 degrees. If we consider the effect of extinction by air mass, the visible magnitudes of the brightest star in DongByeok and Urim are $2.24^{\mathrm{m}}$ and $3.54^{\mathrm{m}}$, respectively. In terms of brightness, the DongByeok might be more adequate objects than Urim as the reference star.

Considering all aforementioned points, although the locations of the Guest Stars in 1073 and 1074 are not described by the nearest constellation to R Aqr, we conclude that the identification of the Guest Stars of 1073 and 1074 with the outbursts of $\mathrm{R}$ Aqr is rather secure.

\section{Implication for R Aqr}

Korean Guest Star records of R Aqr in 1073 and 1074 may provide some helpful hints to understanding of enigmatic R Aqr. First, we estimate two main physical parameters: distance to $\mathrm{R}$ Aqr and the maximum brightness at the outburst of R Aqr. We then explore the implications on the proposed outburst models of R Aqr.

\subsection{Distance and brightness}

Several studies have provided the distance to $\mathrm{R}$ Aqr. Baade (1943, 1944) deduced an expansion age of about 600 years based on a constant expansion rate of outer nebulosity, and suggested a kinematical distance of $260 \mathrm{pc}$. The assumption of the constant expansion rate was based on several observational plates of R Aqr for 16 years. Lépine et al. (1978) on the other hand estimated $181 \mathrm{pc}$, assuming an absolute magnitude for the Mira of $-8.1^{\mathrm{m}}$ at $4 \mu \mathrm{m}$ observation. Solf \& Ulrich (1985) calculated the kinematical distance of $180 \mathrm{pc}$ by observing the equatorial expansion velocity of $55 \mathrm{~km} \mathrm{~s}^{-1}$ and the expansion age of about 640 years. Whitelock (1987) quoted a distance of $250 \mathrm{pc}$ to the R Aqr from GCVS (General Catalogue of Variable Stars). Recent Hipparcos measurement of the distance to the R Aqr is to be in the range of 122 521 pc from the trigonometric parallax of $5.07 \pm 3.15$ milliarcsec (Hipparcos Catalogue 1997).

If we adopt the epoch of outburst to be A.D. 1073, the earlier of the two records, and further assume that the outer nebulosity is expanding at a constant rate (Baade 1943) with the expansion velocity of $55 \mathrm{~km} \mathrm{~s}^{-1}$ for the equatorial outer nebulosity (Solf \& Ulrich 1985), the radius of outer equatorial nebulosity is estimated to be $10826 \mathrm{AU}$. The angular equatorial radius of $42^{\prime \prime}$ (Solf \& Ulrich 1985) corresponds to the distance of R Aqr of 273 pc. This distance falls within the Hipparcos distance range and consistent with the value of Baade and GCVS. If the nebula is decelerating, $273 \mathrm{pc}$ is the lower estimate.

Meanwhile, Henney \& Dyson (1992) suggested a Sedovtype equation of motion for the outer shell-like nebulosity of R Aqr:

$R_{\mathrm{S}}(t)=R_{0}\left(t / t_{0}\right)^{3 / 5}$,

where $R_{\mathrm{S}}$ is the radius of the outer shell, $R_{0}$ the present radius and $t_{0}$ the present time since the outburst. When we adopt the Korean record of 1073 and the current expansion velocity of $55 \mathrm{~km} \mathrm{~s}^{-1}$, Eq. (1) yields a distance of $450 \mathrm{pc}$, marginally consistent with Hipparcos error range, but a little bit too large a distance compared to the previous estimates. This may suggest that the expansion of the outer nebulosity has not been significantly decelerated.

Now, we estimate the brightness of the outburst. The record of 1074 describes the size, i.e. brightness, of the Guest Star as a quince. In order to estimate the brightness of an object described as a quince, we searched in Goryeosa for all astronomical records that had been described to have the size of quince in Goryeosa. We found 94 records: 91 are meteor records, 2 comet records, and one nova record. We estimated the brightness of a quince from the meteor records.

In Goryeosa, there are total of 735 meteor records, and 236 of them have the size information. Meteor is the second most abundant phenomena recorded in Korean history books, and the brightness is described by the size of various objects (Yang et al. 2005). The size of meteors in Goryeosa is described by 8 objects: they are egg, cup, quince, bowl, basin, doe, jar, chopping board, in increasing size, and the rest. Each of them appears in Goryeosa as many as 25, 43, 91, 2, 25, 2, 37, 3, and 8 times, respectively. The remaining 8 records are described in different forms compared to others, and we do not 
consider them. Among meteor records, a quince is the third or fourth smallest object and the most frequent one. Meteor event generally can be seen on the ground when it is brighter than 5 th magnitude. We figure that the record with no description is to be 4 th or 5 th magnitude, the egg $3 \mathrm{rd}$ or 4 th magnitude, the cup 2nd or 3rd magnitude, and so forth in consecutive order. Hence, we estimate that the size of quince corresponds to the apparent magnitude of $1^{\mathrm{m}} \sim 2^{\mathrm{m}}$. If the record of 1074 indeed describes the outburst of $\mathrm{R}$ Aqr with apparent magnitude of $1^{\mathrm{m}} \sim 2^{\mathrm{m}}$, the absolute magnitude of the outburst is estimated to be $M_{\text {outburst }}=-6.2^{\mathrm{m}} \sim-5.2^{\mathrm{m}}$ for the distance of $273 \mathrm{pc}$.

\subsection{Implications for the outburst and nebulosity of $R$ Aqr}

Now we discuss implications for the formative processes of the inner and outer nebulosity. The essential information about R Aquarii is one outburst record in 1073 and another in 1074 , i.e., two outbursts within one year. Moreover, the record of 1074 has additional size information which the record of 1073 does not have. Thus the outburst of 1074 was likely to be brighter than that of 1073 . In addition, no outburst since then has been recorded.

Kafatos \& Michalitsianos (1982) proposed a jet plus accretion disk system produced by the supercritical accretion from the Mira to the hot companion during the periastron passage of highly elliptical orbit to explain the jet observed. They also suggested a nova-like outburst 500 to 1000 years ago to form the extended nebula. Korean records confirm this outburst. However, two successive outbursts within one year interval indicate that the outburst of R Aqr may have been of different nature compared to the usual slow or recurrent nova outburst. The important question is now: can there be an outburst mechanism that produces two successive outbursts with later one being brighter?

Other interesting questions that may be raised are: how are the two outbursts related with the inner and outer nebulosity? It was probably one of the two outbursts or two bursts together that produced the outer nebulosity, which was the original cause to look for the outburst record. But can we rule out the possibility that each burst successively produced each nebula, the inner and outer one? The expansion timescale of the outer nebula is 500 to 1000 years, as previously mentioned. Approximate timescale of the inner nebula, estimated by dividing the angular size of $\sim 13^{\prime \prime}$ times the distance of $273 \mathrm{pc}$ with the expansion velocity of $32 \mathrm{~km} \mathrm{~s}^{-1}$, is about 500 years (Solf \& Ulrich 1985). But the uncertainty of the estimate is quite large, and the timescale as large as one thousand years may not be ruled out. Hence we may imagine that the preburst of 1073 created smaller and slower inner ring-like nebulosity while the main brighter outburst of 1074 produced the larger and faster outer nebulosity in a different direction.

\section{Summary and discussion}

$\mathrm{R}$ Aquarii is known to be a symbiotic binary system and associated with an extended emission nebula with inner and outer shell-like nebulosities. Judging from the angular size of the nebula, the estimated distance, and the expansion velocity, $\mathrm{R}$ Aquarii was suspected to have had an outburst that created current nebulosity within past one thousand years.

Although the Japanese Guest Star record in A.D. 930 was proposed as a candidate outburst (Kafatos \& Michlitsianos 1982), we confirm Li's suspicion that it is a comet record ( $\mathrm{Li}$ 1985; Kanda 1935). Bearing in mind Li's suggestion that a Korean record of A.D. 1073 be the actual record of the outburst of R Aquarii, we searched for and compiled all Guest Star and Peculiar Star records in the three Korean official history books that cover almost two thousand years. We have found two records of Guest Star that may be relevant to R Aquarii. One is the Guest Star record of A.D. 1073 that has been mentioned by $\mathrm{Li}$ (1985). The record reads A Guest Star appears at the south of DongByeok, where DongByeok is the one of 28 oriental constellations. We also have found another Guest Star record in A.D. 1074 that has the exactly the same description, but has an additional brightness description. Both records do not appear in Chinese nor in Japanese records. Moreover, any adequate record to be related with $\mathrm{R}$ Aqr does not appear since then in the Korean history books.

We first examined the positional information in the records. Both records of 1073 and 1074 describe the position as the south of DongByeok. We searched for other possible nova or supernova remnant within $40^{\circ} \times 40^{\circ}$ south of DongByeok. We found none. The probability of having a random nova in the same region for the past 1000 years is roughly $1 / 3$, which is consistent with the records.

Although the actual position of R Aquarii is within other oriental constellation Urim (Yülin in Chinese) rather than DongByeok, we find that it is natural in Korea and China to describe important astronomical phenomena like novae or supernovae with respect to 28 oriental constellations. In addition, stars in DongByeok were more than 1 mag brighter than those in Urim.

By fixing the time of outburst to be A.D. 1073 or 1074, combined with the current nebular size and the expansion velocity, we estimate the distance to R Aquarii to be 273 pc under the assumption of constant expansion. General consistency of this distance with previous estimates suggests that the deceleration of the nebula has not been significant.

The record of 1074 further reads: its size being as large as a quince. By comparing the size of Korean quince against other objects used as a degree of brightness in numerous meteor records, we estimate the apparent magnitude of $\mathrm{R}$ Aquarii at 1074 outburst to be 1 st to 2 nd magnitude. At the distance of $273 \mathrm{pc}$, this gives the absolute magnitude of -6.2 to $-5.2 \mathrm{mag}$ for the outburst.

Analysis of the two records in 1073 and 1074 indicate they are independent and not an erroneous duplicate. This leaves us interesting puzzles. How can there be two nova outbursts separated roughly by one year? It is also possible to interpret existence of brightness information for 1074 outburst while none for 1073 outburst as the former being brighter than the latter. If so, is there an outburst mechanism that produces two successive outbursts with the later one being brighter? How are two outbursts related to two nebulosities, the inner and outer one? 
Table A.1.

\begin{tabular}{ll}
\hline \hline English expression & Original text \\
\hline Baekje & 百濟 \\
Cheonsanyeolchabunyajido & 天象列次分野之圖 \\
DongByeok & 東璧 \\
General Compilation of Chinese Ancient Astronomical Records & 中國古代天象記錄總集 \\
Goguryeo & 高句麗 \\
Goryeo dynasty & 高麗 \\
Goryeosa (The History of Goryeo Dynasty) & 高麗史 \\
Guest Star & 客星 \\
Ichidaiyouki & 一代要記 \\
Japanese Ancient Astronomical Records & 日本天文史料 \\
Jeungbomunheonbigo & 增補文獻備考 \\
Joseon dynasty & 朝鮮 \\
Joseonwangjosillok (The Annals of the Joseon Dynasty) & 朝鮮王朝實錄 \\
Meigetuki & 明月記 \\
Mokgwa & 木瓜 \\
Peculiar Star & 妖星 \\
Samguksagi (The History of the Three Kingdoms) & 三國史記 \\
Sighting of Venus at daylight & 太白書見 \\
Silla & 新羅 \\
Three Domains & 三垣 \\
Three Kingdoms & 三國 \\
Urim & 㹯 \\
28 oriental constellations & 28 宿 \\
\hline & \\
\hline
\end{tabular}

Outburst records of R Aquarii in Korean history books provide the key confirmation on our general understanding of the nature of $\mathrm{R}$ Aquarii and the formation history of its nebula. At the same time, it presents us interesting questions that are needed to be solved for better understanding of R Aquarii.

Acknowledgements. This work is partly supported by Korea Science \& Engineering Foundation through Astrophysical Research Center for the Structure and Evolution of the Cosmos. S.-H. Cho was partially supported by the National Strategic Program of the Ministry of Science and Technology, Korea.

\section{Appendix A: Words in Chinese characters and their English expression (see Table A.1)}

\section{References}

Ahn, S. H., Park, C., \& Yu, K. L. 1996, J. Korean Hist. Sci. Soc., 18, 3

Baade, W. A. 1943, Ann. Report Dir. Mt. Wilson Obs., 1942-1943, 17

Baade, W. A. 1944, Ann. Report Dir. Mt. Wilson Obs., 1943-1944, 12

Beijing Observatory 1988, General compilation of Chinese Ancient Astronomical Records, Jiangsu, PR China

Cho, S.-H., Yang, H.-J., \& Park, C. 1999, Bull. Korean Astron. Soc., 24,45

Clark, D. H., \& Stephenson, F. R. 1977, The Historical Supernova (Oxford, UK: Pergamon)

Chunchugwan (the office for Annals Compilations) 1392-1863, Joseonwangjosillok, The Annals of the Joseon Dynasty

Hipparcos Catalogue 1997, The Hipparcos and Tycho Catalogues,
Harding, K. 1816, Zs. Ap., 1, 449

Henney, W. J., \& Dyson, J. E. 1992, A\&A, 261, 301

Herbig, G. 1980, IAU Circ., 3535

Hollis, J. M., Oliversen, R. J., \& Wagner, R. M. 1989, ApJ, 337, 795

Hsi, T. T. 1958, Smithsonian Contr. Astrophys, 2, 109

Hubble, E. P. 1940, Ann. Report Dir. Mt. Wilson Obs., 1939-1940, 19

Hubble, E. P. 1943, Ann. Report Dir. Mt. Wilson Obs., 1942-1943, 17

Kafatos, M., \& Michalitsianos, A. G. 1982, Nature, 298, 540

Kafatos, M., \& Michalitsianos, A. G. 1983, ApJ, 267, L103

Kanda, S. 1935, Japanese Ancient Astronomical Records, Tokyo, Japan

Kim, B. S., et al. 1145, Samguksagi, The History of the Three Kingdoms

Kim, J. S., et al. 1451, Goryeosa, The History of Goryeo Dynasty

Lampland, C. O. 1923a, Publ. Am. Astron. Soc., 4, 319

Lampland, C. O. 1923b, Publ. Am. Astron. Soc., 4, 365

Lépine, J. R. D., Squeren, A. M., \& Scalise, E. J. 1978, ApJ, 225, 869

Li, J. 1985, Chin. Astron. Astrophys., 9, 322

Park, C. 1998, J. Korean Hist. Sci. Soc., 20, 113

Paresce, F., Albrecht, R., Barbieri, C., et al. 1991, ApJ, 369, L67

Payne-Gaposchkin, C. 1964, The Galactic Novae, Dover, New York, USA

Pskovskii, Y. P. 1972, SvA, 16, 23

Solf, J., \& Ulrich, H. 1985, A\&A, 148, 274

Sopka, R. J., Herbig, G., Kafatos, M., \& Michalitsianos, A. G. 1982, ApJ, 258, L35

Walker, G. 1987, Astronomical Observations (London, UK: Cambridge Univ. Press)

Wallerstein, G., \& Greenstein, J. L. 1980, PASP, 92, 275

Whitelock, P. A. 1987, PASP, 99, 617

Yang, H.-J., Park, C., \& Park, M.-G. 2005, Icarus, in press 